Hideaki Sawai · Akinori Ida • Yuko Nakata

Koji Koyama

\title{
Novel missense mutation resulting in the substitution of tyrosine by cysteine at codon 597 of the type $X$ collagen gene associated with Schmid metaphyseal chondrodysplasia
}

\begin{abstract}
Schmid metaphyseal chondrodysplasia (SMCD) is one of the most common forms of the osteochondrodysplasias. Mutations or deletions in the COL10A1 gene that encodes type $\mathrm{X}$ collagen have been shown to cause this disorder. Most of the gene mutations and deletions are located in the non-collagenous carboxy (C)terminal (NC1) domain. We describe a novel missense mutation in a patient with SMCD that leads to the substitution of Tyr at codon 597 by Cys in the NC1 domain. Sequence analysis indicated that the proband was heterozygous for the mutation. Her parents were homozygous for the normal sequence, indicating the de-novo occurrence of this mutation.
\end{abstract}

Key words Schmid metaphyseal chondrodysplasia (SMCD) - missense mutation - Sequencing - Type X collagen gene · Osteochondrodysplasias

\section{Introduction}

Schmid metaphyseal chondrodysplasia (SMCD) is one of the most common forms of the osteochondrodysplasias. The mode of inheritance is an autosomal dominant trait with complete penetrance. The clinical features of this disorder are short stature with relatively short limbs (but not severe dwarfism), bowed legs, and waddling gait. Facial features and intelligence are normal and life expectancy is not affected. Radiographic features include flaring of the metaphyses, a wide, irregular growth plate in the distal femur, and coxa vara (Lachman et al. 1988).

Gene mutations or deletions of the COL10A1 gene that encodes type $\mathrm{X}$ collagen have been shown to cause this

H. Sawai · A. Ida $\cdot$ Y. Nakata $\cdot$ K. Koyama $(\bowtie)$

Department of Obstetrics and Gynecology, Hyogo College of Medicine, 1-1 Mukogawa-cho, Nishinomiya-shi, Hyogo 663-8131, Japan

Tel. +81-798-45-6481; Fax +81-798-46-4163

e-mail: kkoyama@hyo-med.ac.jp disorder. A 13-bp deletion in the region of the noncollagenous carboxy (C)-terminal (NC1) domain of type X collagen was identified in one allele segregating with autosomal dominant SMCD (Warman et al. 1993). Subsequently, additional mutations, i.e., frameshift and missense mutations, were reported (McIntosh et al. 1994, Wallis et al. 1994). We report a novel gene mutation in the NC1 domain of type X collagen in a Japanese patient with SMCD.

\section{Patient and methods}

Patient

The proband first presented in 1976 at the age of 3 years with relatively short limbs, waddling gait, and genu varum. Radiographic features included a shortening of tubular bones, bowed legs, and bilateral coxa vara. Her parents were not affected. The clinical diagnosis was a sporadic form of SMCD. She had bilateral osteotomies, performed twice, to correct the bent, short femurs. When she presented at our hospital, she was 22 years of age and was of short stature $(143 \mathrm{~cm})$. Although her lower limbs were short, by this time the abnormal gait had been considerably corrected.

PCR and sequencing

Genomic DNA was isolated from blood samples of the proband and her parents, using a blood and cell culture DNA mini kit (Qiagen, Hilden, Germany). The NC1 domain of collagen $\mathrm{X}$ was amplified by polymerase chain reaction (PCR), using the primers $\mathrm{H} 175^{\prime}$ GCCTGTGTCTGCTTTTACTG-3' and H23 5'TTCAGCCTACCTCCATATGC-3' (McIntosh et al. 1994), and AmpliTaq Gold supplied with a GeneAmp kit (PE Applied Biosystems, Norwalk, CT, USA). The PCR conditions were $95^{\circ} \mathrm{C}$ for $30 \mathrm{~s}, 62^{\circ} \mathrm{C}$ for $30 \mathrm{~s}$, and $72^{\circ} \mathrm{C}$ for $30 \mathrm{~s}$ for 30 cycles with the GeneAmp PCR System 9700 (PE Applied Biosystems). 
Direct sequencing of the amplified fragment was performed using a Dye Terminator Cycle Sequencing FS Ready Reaction Kit and DNA Sequencer ABI PRISM 373A (PE Applied Biosystems). To confirm the missense mutation of the proband, the amplified fragments were cloned into pT7Blue T-Vector (Novagen, Madison, WI, USA) and then subjected to sequencing.

\section{Results and discussion}

A 490-bp fragment was amplified by PCR (data not shown). Sequence analysis indicated that the proband was heterozygous for the mutation of the nucleotide at position 1790 (nucleotides numbered according to Thomas et al. [1991]) (Fig. 1). A single-base-pair mutation, of $A$ to $G$, at nucleotide 1790 resulted in the substitution of Tyr by Cys at codon 597 (Y597C). Her parents were homozygous for the normal sequence at nucleotide 1790 , indicating the de-novo occurrence of this mutation. The other sequence of the proband was normal. This heterozygous mutation was confirmed by sequence analysis of the cloned PCR products (data not shown).

Amino acid substitution at codon 597 has been reported previously, by Bonaventure et al. (1995); however, that mutation occurred at nucleotide 1789, resulting in the substitution of Tyr by His (Y597H). They also reported a Glyto-Glu substitution at codon 595 (G595E). In the neighboring region at codon 598 another mutation (Y598D), in which Tyr was replaced by Asp, was reported by (Wallis et al. (1994). The substitution of Cys by Arg at residue 591 (C591R) reported by McIntosh et al. (1994) was also located in this neighboring region.

McIntosh et al. (1995) noted that most of the mutations and deletions were located in the NC1 domain. They suggested that the concentration of mutations within this domain was consistent with the hypothesis that the phenotype

(a)
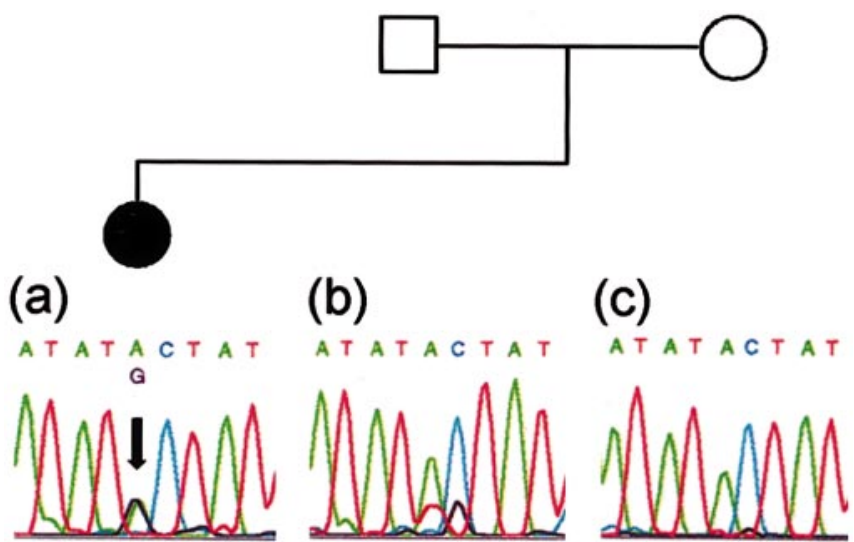

Fig. 1 Direct sequencing of polymerase chain reaction amplified genomic DNA from the proband (a), father (b), and mother (c). A heterozygous single-base-pair transition from $A$ to $G$ at nucleotide 1790 (arrow) was detected in the proband. This mutation caused substitution of a tyrosine residue by cysteine at codon 597. No such change was detected in the proband's father or mother was the result of a reduction in the level of mature type $\mathrm{X}$ collagen. This was based on the belief that: (1) this domain was involved in the initiation of collagen trimerization, and (2) mutant domains interfered with this trimerization (Chan et al. 1995). However, of the five different mutations observed in Japanese patients to date, two were located in the N-terminal domain (Pokharel et al. 1995; Matsui et al. 1996; Ikegawa et al. 1997). This suggested that the N-terminal domain may also play a crucial role in the formation of type X collagen (Ikegawa et al. 1997). Recently, Chan et al. (1998) reported that, in a patient with SMCD, no mutant mRNA or mutant protein was detected where a single nucleotide substitution resulted in the Tyr632 codon being changed to a stop codon. Considering that mutations of type X collagen have not been detected in all SMCD patients (Bonaventure et al. 1995; Ikegawa et al. 1997), these results suggest that haplo-insufficiency of type $\mathrm{X}$ collagen may be the principal cause of SMCD, regardless of the mechanism leading to the shortage of type X collagen.

Acknowledgements The authors would like to thank Gregory $\mathrm{H}$. Smith for helping in the preparation of this manuscript.

\section{References}

Bonaventure J, Chaminade F, Maroteaux P (1995) Mutations in three subdomains of the carboxyl-terminal region of collagen type $\mathrm{X}$ account for most of the Schmid metaphyseal dysplasia. Hum Genet 96: $58-64$

Chan D, Cole WG, Rogers JG, Bateman JF (1995) Type X collagen multimer assembly in vitro is prevented by a Gly ${ }^{618}$ to Val mutation in the $\alpha 1(\mathrm{X}) \mathrm{NC1}$ domain resulting in Schmid metaphyseal chondrodysplasia. J Biol Chem 270: 4558-4562

Chan D, Weng YM, Graham HK, Sillence DO, Bateman JF (1998) A nonsense mutation in the carboxyl-terminal domain of type $\mathrm{X}$ collagen causes haploinsufficiency in Schmid metaphyseal chondrodysplasia. J Clin Invest 101: 1490-1449

Ikegawa S, Nakamura K, Nagano A, Haga N, Nakamura Y (1997) Mutations in the N-terminal globular domain of the type $\mathrm{X}$ collagen gene (COL10A1) in patients with Schmid metaphyseal chondrodysplasia. Hum Mutat 9: 131-135

Lachman RS, Rimoin DL, Spranger L (1988) Metaphyseal chondrodysplasia, Schmid type: Clinical and radiographic delineation with a review of the literature. Pediatr Radiol 18: 93-102

Matsui Y, Kimura T, Tsumaki N, Yasui N, Ochi T (1996) A recurrent 199delCT mutation of the type X collagen gene in a Japanese patient with Schmid metaphyseal chondrodysplasia. Jpn J Hum Genet 41: 339-342

McIntosh I, Abbott MH, Warman ML, Olsen BR, Francomano CA (1994) Additional mutations of type X collagen confirm COL10A1 as the Schmid metaphyseal chondrodysplasia locus. Hum Mol Genet 3: 303-307

McIntosh I, Abbott MH, Francomano CA (1995) Concentration of mutations causing Schmid metaphyseal chondrodysplasia in the Cterminal noncollagenous domain of type X collagen. Hum Mutat 5: 121-125

Pokharel RK, Alimsardjono H, Uno K, Fujii S, Shiba R, Matsuo M (1995) A novel mutation substituting tryptophan with arginine in the carboxyl-terminal, non-collagenous domain of collagen $\mathrm{X}$ in a case of Schmid metaphyseal chondrodysplasia. Biochem Biophys Res Commun 217: 1157-1162

Thomas JT, Cresswell CJ, Rash B, Nicolai H, Jones T, Solomon E, Grant ME, Boot-Handford RP (1991) The human collagen X gene: Complete primary translated sequence and chromosomal localization. Biochem J 280: 617-623

Wallis GA, Rash B, Sweetman WA, Thomas JT, Super M, Evans G, Grant ME, Boot-Handford RP (1994) Amino acid substitutions of 
conserved residues in the carboxyl-terminal domain of the $\alpha 1(\mathrm{X})$ chain of type $\mathrm{X}$ collagen occur in two unrelated families with metaphyseal chondrodysplasia type Schmid. Am J Hum Genet 54: 169-178
Warman ML, Abbott M, Apte SS, Hefferon T, McIntosh I, Cohn DH, Hecht JT, Olsen BR, Francomano CA (1993) A type X collagen mutation causes Schmid metaphyseal chondrodysplasia. Nature Genet 5: 79-82 